\title{
Targeting triple-negative breast cancer
}

Targeted drugs have shown considerable success in the treatment of hormone-receptor-positive and ERBB2-overexpressing breast cancers. However, no such agents are available for breast cancers lacking these characteristics - known as 'triple-negative' - which represent $\sim 15 \%$ of breast cancers. A report in the Proceedings of the National Academy of Sciences USA now provides in vivo evidence that inhibitors of poly(ADP-ribose) polymerase 1 (PARP1), an enzyme that has a key role in DNA repair, might become effective targeted therapies for such cancers.

Triple-negative breast cancers are often characterized by defects in DNA repair mechanisms involving homologous recombination; for example, owing to dysfunction of the breast cancer type 1 susceptibility protein (BRCA1). Indeed, mutations in BRCA1 are known to predispose individuals to hereditary breast and ovarian cancers. So, as BRCA-deficient cells had previously been shown to be highly sensitive to the effects of inhibiting PARP1, Rottenberg and colleagues investigated the effects of the PARP1 inhibitor AZD2281 in a mouse model of breast cancer.

To gain a more realistic understanding of the therapeutic potential of AZD2281, the authors chose a model that was based on genetically engineered mice in which $\mathrm{BrCa}^{-/-} p 53^{-/-}$mammary tumours arise spontaneously. This model recapitulates several key features of human BRCA1-associated breast cancers - such as lack of hormone receptor and ERBB2 expression, and a high degree of genomic instability. Moreover, this model seems to mimic clinical responses to anticancer drugs more effectively than traditional xenografts in immunodeficient mice.

Treatment of tumour-bearing mice with AZD2281 inhibited tumour growth without obvious signs of toxicity, and a 100-day schedule increased median survival after treatment initiation from 10 days to 131 days. However, long-term treatment resulted in the emergence of drug resistance, which the authors showed to be due to the upregulation of genes encoding drug-efflux transporters known as P-glycoproteins. This resistance could be reversed by combining AZD2281 with tariquidar, a specific inhibitor of P-glycoprotein.

Finally, as inhibition of PARP1 has been reported to enhance the effects of DNA-damaging drugs, such as those in the platin class, the authors evaluated AZD2281 together with either cisplatin or carboplatin. Combination therapy increased recurrence-free and overall survival compared with platin monotherapy, although increased toxicity was observed with the platin combination.

Data from Phase I trials of AZD2281 so far indicate a favourable toxicity profile and objective responses in patients with BRCA1associated cancer. As the authors highlight, genetically engineered mouse models, such as the one

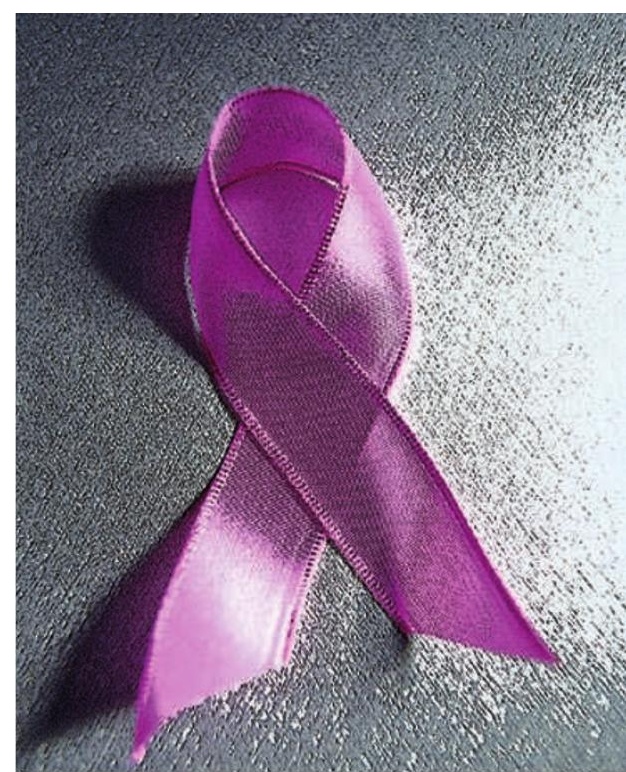

described in this paper, could be valuable in optimizing PARP1 inhibitor combinations with other drugs for investigation in further clinical trials.

Peter Kirkpatrick

ORIGINAL RESEARCH PAPER Rottenberg, S. et al. High sensitivity of BRCA1-deficient mammary tumors to the PARP inhibitor AZD2281 alone and in combination with platinum drugs. Proc. Natl Acad. Sci. USA 105, 17079-17084 (2008) FURTHER READING Liu, X. et al. Somatic loss of BRCA1 and $p 53$ in mice induces mammary tumors with features of human BRCA1-mutated basal-like breast cancer. Proc. Natl Acad. Sci. USA 104, 12111-12116 (2007)|Rottenberg, S. et al.

Selective induction of chemotherapy resistance of mammary tumors in a conditional mouse model for hereditary breast cancer. Proc. Natl Acad. Sci. USA 104, 12117-12122 (2007)| Sharpless, N. E. \& DePinho, R. A. The mighty mouse: genetically engineered mouse models in cancer drug development. Nature Rev. Drug Discov. 5, 741-754 (2006) 